\title{
MODELLING OF A STOCHASTIC CONTINUOUS SYSTEM ${ }^{1}$
}

\author{
Martin Albertyn ${ }^{2}$ \\ Department of Industrial and Systems Engineering \\ University of Pretoria \\ Pretoria, South Africa \\ Paul S Kruger \\ Department of Industrial and Systems Engineering \\ University of Pretoria \\ Pretoria, South Africa
}

\section{KEYWORDS}

Simulation model, Continuous model, Stochastic model, Deterministic model, Continuous process plant.

\begin{abstract}
The key objective is to develop a method which can be utilized to model a stochastic continuous system. A system from the "real world" is used as the basis for the simulation modelling technique that is presented. The conceptualization phase indicates that the model has to incorporate stochastic and deterministic elements. A method is developed that utilizes the discrete simulation ability of a stochastic package (ARENA), in conjunction with a deterministic package (FORTRAN), to model the continuous system. (Software packages tend to specialize in either stochastic, or deterministic modelling.) The length of the iteration time interval and adequate sample size are investigated. The method is authenticated by the verification and validation of the defined model. Two scenarios are modelled and the results are discussed. Conclusions are presented and strengths and weaknesses of this method are considered and discussed.
\end{abstract}

${ }^{1}$ Presented at the $11^{\text {th }}$ European Simulation Multiconference (ESM'97) in Istanbul. Turkey (1-4 June 1997) and included in the conference proceedings.

2Currently in the employ of Gennan, a Business Unit of Dendex, a Division of Denel. P O Box 7063, 0001 Pretoria, South Africa. (E-mail: martin(dendex.denel.co.za) 


\section{INTRODUCTION}

The system which is used as the basis for the simulation modelling technique illustrated in this paper, is a continuous process plant. The plant produces chemical products from coal. A simplified schematic representation of the plant is shown in figure 1: "Continuous process plant"[4]. The plant is representative of a real continuous process plant (and also of any stochastic continuous system with complex interrelationships). Changes to the original data are incorporated to protect client confidentiality.

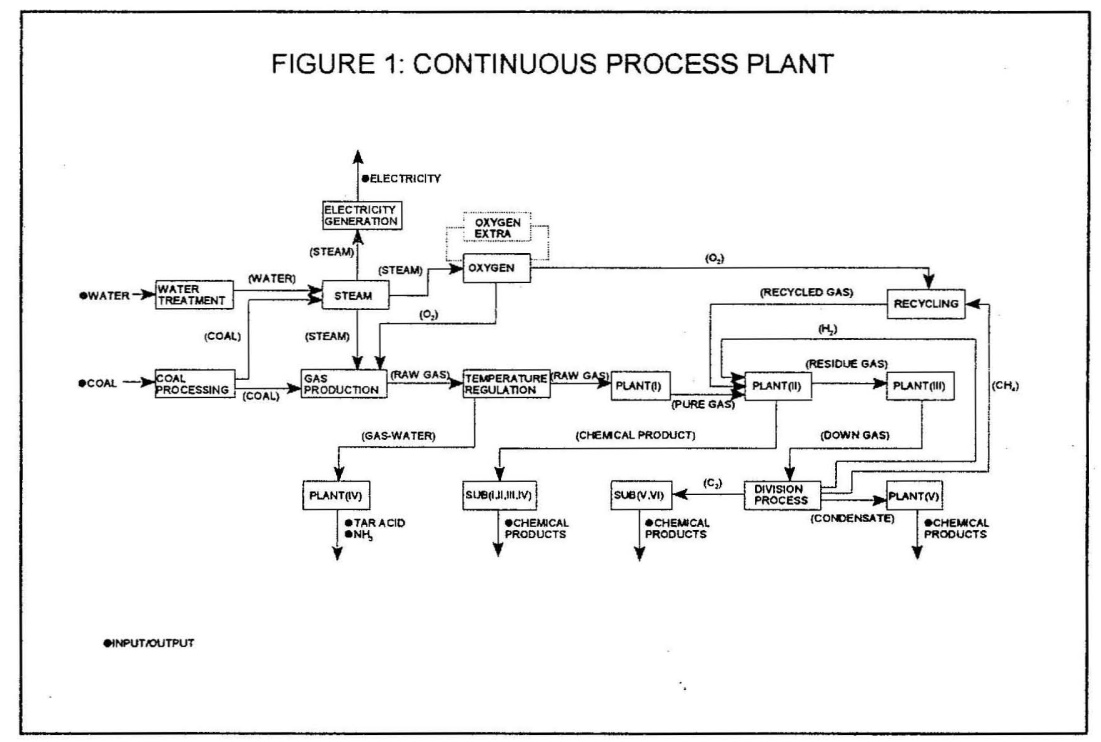

The original need for a simulation model of the plant arose, because the plant management identified the necessity of a decision support tool at a strategic level. In a plant of this size and complexity it is extremely difficult to predict what the effect of proposed changes is going to be on the operation of the plant. The complex interrelationships that define the operation of the plant, as well as random events such as failures of equipment can be dealt with in a simulation model.

The main objectives of the model are:

a) To identify problem areas ("bottlenecks") in the system.

b) To study the effect of projected scenarios on the system. Projected scenarios may include added capacity at bottlenecks, changes in the support philosophy, etc. (In this paper the specific scenario that is under scrutiny, is the effect of an extra Oxygen train on the continuous process plant.) 


\section{PROBLEM DEFINITION [4]}

To demarcate the problem it is necessary to define the system being considered. The composition and process logic of the plant can be derived from figure 1 . For the purpose of the model, the total plant is considered to consist of 20 smaller plants and 148 modules. A module can be seen as a group of equipment that has a specific function, for example, in the Gas production plant the coal is processed by a gas production unit. There are a number of gas production units, each consisting of many components. For the resolution of this model each individual gas production unit is taken as being a module. Some plants consist of groups of different types of modules. Parallel lines of modules in a plant are referred to as "trains". The plant has a complex switching capability. This implies that if one of the modules of a train goes down, the whole train is not necessarily rendered inoperative.

The throughput of the total plant depends on the capacities and the availabilities of the different modules. The availability of a module depends on the service schedule and the occurrence of failures. Services are chronological, scheduled events and failures are stochastic events.

\section{MODEL CONCEPTUALIZATION [4]}

The following key characteristics can be identified:

a) It is a "continuous flow" problem.

b) Some elements of the problem has a stochastic nature.

c) The problem (plant) possesses complex interrelationships.

In the "real world" the "time between failures" and the "time to repair" are stochastic events. This is in contrast to services which start at predetermined times by decommissioning of the module for a predetermined time interval. The complex interrelationships are manifested in both the composition and the operation of the plant. The plant does not have a simple serial or parallel configuration. The switched on or off logic of the different modules also presents complex interrelationships from an operational point of view. The continuous nature of the plant implies that all 148 modules are in a way intrinsically interlinked. A breakdown in production (caused by service or failure) at one point, does have an immediate effect on upstream and downstream operations.

In classical mathematics the behaviour of a continuous system is usually modelled with the help of differential equations. Analytical solutions can, however, only be found for rather simplistic systems. As soon as more complex differential equations are encountered, numerical methods seem to be the only viable solution. One such method involves the discretisation (division into discrete elements) of the continuous behaviour of a system into behaviour at specific intervals in time. The state of the system is calculated at every time interval. The total behaviour of the system is then found by the summation of the behaviour at the different points in time. If the time interval between evaluations is chosen in accordance to the dynamic response characteristics of the system, the results obtained can be a very close approximation of the real process. It stands to reason that a continuous flow process plant can also be modelled in this way. 
Throughput can be defined by the following equation:

\section{Throughput $=($ Capacity $)($ Availability $)($ units/time $)$}

Where capacity is a function of composition and process-capacity and availability is a function of services and failures. Throughput may vary over time, as it is derived from time dependent elements.

To model throughput at any given time, the status of each individual module must be known. The model needs to identify the module group that represents the momentary "bottleneck" in the system at that specific moment in time. This point is the momentary "driver" that will determine the maximum possible throughput. From this point the switched on or offlogic, for upstream and downstream module groups, can be determined. The philosophy of maximum possible throughput will lead to all the modules in the identified "bottleneck" module group being switched on. In contrast to this, the upstream and downstream module groups could have some modules that are not switched on. Due to the complex interrelationship characteristic of the plant, the identification of the momentary "bottleneck" and the determination of the associated switched on or off logic, require complex mathematical calculations. With the module status known, the momentary "bottleneck" identified, and the switched on or off logic determined, the throughput can be calculated.

To summarize:

a) It seems that to model the system, stochastic and deterministic elements must be incorporated.

b) The service schedules and service durations are strictly chronological events and thus can be handled in a deterministic manner.

c) The failures and repair times are stochastic by nature.

d) For the switched on or off logic complex deterministic calculations are required. (The switched on or off logic represents the control of the process.)

e) Furthermore, to identify the "bottlenecks", it is necessary to keep records of certain parameters over the period under consideration (counters and statistics).

f) If all of the previously mentioned procedures are included in a model and evaluated at predetermined time intervals, the results should approximate the operation of the real plant.

A graphical representation of this concept is shown in figure 2: "Conceptualized model". 


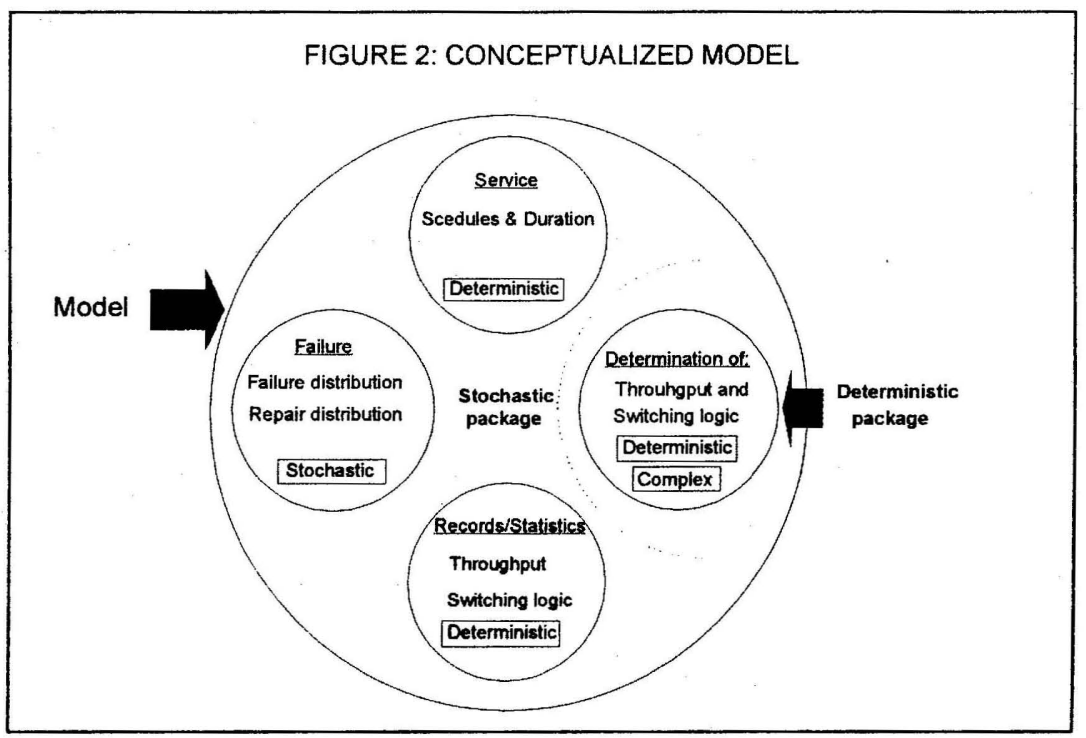

Figure 2 Conceptualized model

Most of the simulation software packages available, have the ability to facilitate the majority of the elements defined in figure 2. Software packages, however, tend to specialize in either stochastic or deterministic modelling, but packages that are aimed at industrial modelling, always include stochastic capability. The problem is that these packages are often cumbersome when complex deterministic calculations are to be addressed. This modelling approach thus requires a specialized, stochastic package to work in conjunction with a specialized, deterministic software package. The three elements on the left hand side of figure 2 can then be accommodated by the stochastic package, while the switched on or off logic and throughput calculations can be handled by the deterministic package. This modelling approach has the advantage that it uses the strong points of both stochastic packages and deterministic packages in an integrated approach.

\section{MODEL CONSTRUCTION}

An ARENA model is used to accommodate the three elements on the left hand side of figure 2 . (ARENA is a simulation software package from Systems Modeling Corporation). Statistical distributions are used to model the stochastic elements [2]. The ARENA model incorporates a FORTRAN subroutine that handle the complex deterministic calculations A novel feature of the ARENA model is that it is generic in terms of modules, module groups and plants, thus facilitating easy implementation of changes and further developments.

Survey results indicates the growing importance of "user-friendliness" of software [3]. Seen in this perspective, some of the inputs to the model are handled with the help of separate input files. 


\section{DETERMINATION OF THE ITERATION TIME INTERVAL AND ADEQUATE SAMPLE SIZE $[1,2,4]$}

In the previous section it is indicated that the behaviour of a continuous system over time can be modelled by discretisation of the continuous behaviour into behaviour at specific intervals in time. The total behaviour of the system is found by the summation of the behaviour at the different points in time. If the time interval between evaluations is chosen in accordance with the dynamic response characteristics of the system, the results obtained can be a very close approximation of the real process.

Investigation of the processes involved in the continuous process plant, suggests that an iteration time interval of one (1) hour should be appropriate. The validity of this assumption is tested by starting with a very short iteration time interval and increasing it until the answers start to deviate from the perceived correct one. In this instance, the perceived correct answer will be the one generated with the shortest iteration time interval. The results indicate that if a deviation of $1 \%$ is taken as an acceptable deviation, all iteration time intervals up to and including six (6) hours seem acceptable. The assumption of using a one (1) hour iteration time interval thus seems to be valid.

The results from a stochastic model may show slight variation between different replications, because of the randomness of the chance events such as breakage. This implies that during the simulation run of a scenario, more than one replication has to be completed in order to find answers that are "stabilized" and representative of the simulated scenario. The minimum sample size that is needed to get a mean value that is representative of the simulated scenario, can be calculated with the help of an equation from Crow [1]. A sample size of five (5) replications is found to be sufficient in this instance to accept the mean values derived from the five (5) replications, as being representative of the simulated scenario.

\section{MODEL VERIFICATION AND VALIDATION}

The verification involved the checking of the operation of the logic (FORTRAN subroutine) in terms of the chemical processes involved and the working of the ARENA part of the program in terms of the services and breakages. The number of services and breakages generated were counted and evaluated against their "real world" values.

The simulation result (validated against the output from Gas production) deviates only $0,59 \%$ from the "real world" value for a known scenario. This small percentage can for all practical purposes being taken as zero. It can therefore be accepted with a high level of confidence, that the model is a valid representation of the real continuous process plant [4]. 


\section{MODEL RESULTS [4]}

To reach the main objectives that are stated, two simulation runs were completed. The first run (RUN1) utilizes the "standard" model, that is the continuous process plant without the addition of the extra Oxygen train. The second run (RUN2) includes the additional Oxygen train. Each of the simulation runs comprised of ten (10) replications, even though five (5) replications would have been adequate in this instance (as indicated by Crow). The period of time simulated in both RUN1 and RUN2 is 18 months.

One method of identification of "bottlenecks" in a system, is to identify the module groups which are highly utilized. The results from RUN1 show that the "bottleneck" plants are Plant(II)-A $(94,3 \%)$, Plant(I) $(94,1 \%)$, Oxygen-A (91,3\%), Oxygen-B $(89,6 \%)$, Gas production $(86,7 \%)$, Plant(III) $(85,5 \%)$, Division process $(85,5 \%)$ and Temperature regulation $(81,1 \%)$. It should be noted that Plant(II) consists of two different types of modules (designated Plant(II)-A and Plant(II)-B) and Oxygen consists of three different types of modules (Designated Oxygen-A, Oxygen-B and Oxygen-C). The plants are prioritized in terms of decreasing importance with an $80,0 \%$ utilization level as the cut-off point for "bottleneck" identification. This result correlates closely with the "real world" experience of the plant operators where the Plant(II)-A and Plant(I) plants have the reputation of being the "troublemakers" of the total plant.

The results from RUN2 show that the utilization levels of all the plants (except Oxygen) show a slight increase in the RUN2 scenario, from the values generated by the RUN1 scenario.. The RUN2 "bottleneck" plants are Plant(II)-A (95,6\%), Plant(I) (95,2\%), Gas production (87,9\%), Division process $(86,8 \%)$, Plant(III) $(86,3 \%)$ and Temperature regulation $(82,2 \%)$. A comparison indicates that Oxygen-A and Oxygen-B, which are featured prominently in the RUN1 results, are not part of the prioritized "bottlenecks" any more in the RUN2 scenario. In the RUN2 scenario the total Oxygen plant (inclusive of the additional Oxygen train), does not qualify as a priority "bottleneck" any more.

A comparison of the output of Gas production shows an increase from RUN1 to RUN2. The Gas production output and above-mentioned behaviour of the utilization levels, are an indication that the addition of the extra Oxygen train results in an increase in the throughput of the continuous process plant. The increase in the output from Gas production from RUN1 to RUN2 is $1,43 \%$. It is equal to an increase in the yearly production of Raw gas from Gas production of 5,15 production days. Thus 5,15 additional production days a year (in terms of RUN1 production days) are provided, if the RUN1 scenario is compared with the RUN2 scenario. 


\section{STRENGTHS OF THE METHOD}

a) The construction of the ARENA part of the model is strictly generic in terms of plants and modules.

b) All the software involved is capable of operating on an IBM compatible personal computer.

c) Both ARENA and FORTRAN are standard and easily obtainable software packages.

d) ARENA makes provision for the incorporation of stochastic phenomena like failures and repair times into the model, with the use of different intrinsic mathematical distributions.

e) The method allows the modeller to incorporate complex decision-making processes into the model by virtue of the inclusion of FORTRAN.

f) "User-friendliness" is enhanced by the use of input files.

g) The incorporation of FORTRAN into the model to handle the complex mathematical calculations that are required, assists in keeping simulation runtimes within acceptable limits.

\section{WEAKNESSES OF THE METHOD}

a) The FORTRAN subroutine has complex structures and to a large extent it is not generic.

b) The method gives rise to a complicated structure, involving two different software packages and complex interfacing, compiling and linking.

c) The complex structure and stochastic nature of the model complicates debugging. 


\section{CONCLUSIONS}

It can be accepted with a high level of confidence (proved through verification and validation), that the model is a valid representation of the real continuous process plant. The method demonstrated in this paper to model a stochastic continuous system, can thus be accepted as an authentic and valid method of modelling. Even though the model is only utilized in this paper to reach the objectives that are stated, it can be implemented with confidence in any further projected scenario analysis and "what if" studies.

There is a growing importance of "user-friendliness" in simulation modelling. Clients in the simulation fraternity do not only want the answers to a few pre-selected scenarios; they want access to the answers of "what if" questions on a daily basis. Through the use of input files the method that is demonstrated in this paper, already aims to satisfy this requirement. Although ARENA is an expensive simulation package, a so-called "runtime" version is available at a much more reasonable price. This version allows the user (client) to realize changes in the governing parameters of the model, but not in the structure of the model. This gives the client access to a simple modelling capability, once the model has been developed by a model developer. At present the simulation model of the continuous process plant is running on a "runtime" version of ARENA at the plant.

This indicates a future where the advent of "runtime" versions, will allow the user (client) closer involvement with the simulation model and a limited modelling capability. Such developments will lead to higher levels of acceptance by the management of industry, of simulation modelling techniques as valid decision-making support tools.

\section{REFERENCES}

1. CROW, E.L.; F.A.DAVIS; and M.W.MAXFIELD. 1960. Statistics Manual. Dover Publications Inc., New York, 48.

2. PEGDEN, C.D.; R.E.SHANNON; and R.P.SADOWSKI. 1990. Introduction to Simulation Using SIMAN. McGraw-Hill Inc., New York, 46-51.

3. Simulation Fax Survey Results. 1993. Industrial Engineering, May 1993, vol.25, no.5, 10.

4. ALBERTYN M; 1995. Modelling of a stochastic contimuous system. Unpublished Masters thesis, Department of Industrial and Systems Engineering, University of Pretoria.

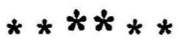

